\title{
Fasting proinsulin levels are significantly associated with 20 year cancer mortality rates. The Hoorn Study
}

\author{
I. Walraven - E. van 't Riet • C. D. A. Stehouwer • \\ B. C. P. Polak • A. C. Moll • J. M. Dekker • G. Nijpels
}

Received: 7 December 2012 / Accepted: 24 January 2013 /Published online: 5 March 2013

(C) Springer-Verlag Berlin Heidelberg 2013

\begin{abstract}
Aims/hypothesis Proinsulin is possibly associated with cancer through activation of insulin receptor isoform A. We sought to investigate the associations between proinsulin and 20 year cancer mortality rates.

Methods The study was performed within the Hoorn Study, a population-based study of glucose metabolism in individuals aged $50-75$ years in the Dutch population. Fasting proinsulin levels were measured twice by a doubleantibody radioimmunoassay. Participants were continuously followed to register mortality; causes of death were derived from medical records. Cox survival analyses were performed to assess the 20 year risk of death from cancer in relation to proinsulin. All analyses were adjusted for age and sex, with additional adjustments for traditional risk factors. The effect modification of glucose metabolism and sex was tested.
\end{abstract}

I. Walraven $(\varangle) \cdot$ E. van 't Riet • B. C. P. Polak · A. C. Moll • J. M. Dekker · G. Nijpels

EMGO Institute for Health and Care Research,

Van der Boechorststraat 7,

1081 BT, Amsterdam, the Netherlands

e-mail: i.walraven@vumc.nl

I. Walraven • B. C. P. Polak • A. C. Moll

Department of Ophthalmology, VU University Medical Center,

Amsterdam, the Netherlands

I. Walraven · E. van 't Riet · J. M. Dekker

Department of Epidemiology and Biostatistics,

VU University Medical Center, Amsterdam, the Netherlands

C. D. A. Stehouwer

Department of Internal Medicine and Cardiovascular Research Institute Maastricht (CARIM), Maastricht University Medical

Center, Maastricht, the Netherlands

G. Nijpels

Department of General Practice, VU University Medical Center, Amsterdam, the Netherlands
Results Proinsulin levels were measured in 438 individuals (41\% normal glucose tolerance, $35.7 \%$ impaired glucose metabolism, $23.3 \%$ type 2 diabetes). Of these participants, 53 died from cancer. After adjustment for age and sex, proinsulin $>16.5 \mathrm{pmol} / 1$ (the upper tertile) was significantly associated with a twofold risk of cancer mortality (HR 2.01, $95 \%$ CI 1.16, 3.46) compared with individuals with lower proinsulin levels. Additional adjustment for glucose metabolism, BMI and smoking did not substantially change the results (HR 1.91, 95\% CI 1.04, 3.52). No interaction with glucose metabolism or sex was observed.

Conclusions/interpretation Individuals with fasting proinsulin levels $>16.5 \mathrm{pmol} / 1$ have a twofold risk of cancer mortality over a 20 year time span. These findings provide population-based evidence for the independent association between high proinsulin levels and cancer mortality rates.

Keywords Cancer · Cancer mortality · Epidemiology · Glucose metabolism · Proinsulin · Type 2 diabetes

\begin{tabular}{ll}
\multicolumn{2}{l}{ Abbreviations } \\
FPG & Fasting plasma glucose \\
HOMA-IR & HOMA of insulin resistance \\
ICD & International Classification of Diseases \\
IGM & Impaired glucose metabolism \\
IR-A & Insulin receptor A \\
IR-B & Insulin receptor B \\
-2LL & -2 Log likelihood \\
NGM & Normal glucose metabolism
\end{tabular}

\section{Introduction}

Individuals with impaired glucose metabolism (IGM) or type 2 diabetes might have an increased risk of cancer and cancerrelated mortality [1-7]. Insulin resistance, hyperglycaemia, 
high levels of insulin, and production of IGF-I and IGF-II have been suggested to induce tumour cell growth, thereby possibly providing a mechanistic link between glucose metabolism and cancer $[1,3,6,8]$.

Proinsulin is a precursor of insulin and is co-secreted proportionally to insulin by the pancreatic beta cell [9]. Increased levels of proinsulin in the circulation are considered to be a sign of defective proinsulin-to-insulin conversion and may reflect beta cell dysfunction [10-12]. Indeed, hyperproinsulinaemia in non-diabetic individuals is known to be highly predictive of type 2 diabetes [11, 13]. An in vitro study by Malaguarnera et al [14] recently showed that proinsulin binds with and activates insulin receptor isoforms, in particular insulin receptor A (IR-A). IR-A mediates effects on growth and survival [15-17] and is overexpressed in several types of cancer, for example thyroid cancer [16].

None of the studies [1-7] that reported on the association between glucose metabolism and cancer took proinsulin into account. In the light of recent evidence [14] for the potential link between proinsulin and cancer, proinsulin could explain part of the overall risk of developing cancer. To our knowledge, no other study has addressed the association between proinsulin and cancer mortality rates. Such data are needed, since they might contribute to knowledge of the mechanisms involved. The Hoorn Study is a population-based cohort study with a long-term and precise follow-up of cause-related death. In a subsample of the Hoorn Study cohort, proinsulin concentrations were determined on two separate days at baseline in 1989 [11]. This paper reports on the association between proinsulin and cancer mortality.

\section{Methods}

Study population This study was conducted within the Hoorn Study, a population-based cohort study of type 2 diabetes in the Dutch population, which started in 1989 and had 2,484 participants [18]. More extensive measurements were performed in a stratified random sample of the Hoorn Study $(n=631)$ [19]. Insulin and proinsulin levels were measured in individuals with normal glucose metabolism (NGM) and IGM, and in newly diagnosed type 2 diabetes patients not using glucose-lowering therapy. Participants with a verified history of type 2 diabetes were excluded from proinsulin measurements $(n=56)$ [20]. Furthermore, we excluded participants who, for technical reasons $(n=63)$ or because of missing samples $(n=74)$, did not have proinsulin values at one or both of the oral glucose tolerance tests. This left a final cohort size of 438 individuals. Written informed consent was obtained from all participants. The Ethical Review Committee of the VU University Medical Center (Amsterdam, the Netherlands) approved the Hoorn Study.
Measurements Anthropometric measurements were obtained from all participants. Weight, height, and hip and waist circumferences were measured with participants barefooted and wearing light clothes. Blood pressure was measured on the right upper arm after $5 \mathrm{~min}$ of rest, using a random-zero sphygmomanometer (Hawksley-Gelman, Lancing, UK). Mean blood pressure was then calculated as the mean of two measurements [18].

Fasting plasma glucose (FPG) concentrations and $2 \mathrm{~h}$ postload plasma glucose concentrations were determined by the glucose dehydrogenase method (Merck, Darmstadt, Germany). For the present analyses, we applied the American Diabetes Association [21] diagnostic criteria of 2011 for [1] type 2 diabetes (i.e. $\mathrm{HbA}_{1 \mathrm{c}} \geq 6.5 \% /[48 \mathrm{mmol} / \mathrm{mol}]$ and/or $\mathrm{FPG} \geq 7.0 \mathrm{mmol} / 1$ and/or $2 \mathrm{~h}$ plasma glucose $\geq 11.1 \mathrm{mmol} / \mathrm{l}$ ); [2] pre-diabetes (non-diabetic levels for all three markers of hyperglycaemia and at least one of the following in the indicated ranges: $\mathrm{HbA}_{1 \mathrm{c}} 5.7-6.4 \%$ [39$48 \mathrm{mmol} / \mathrm{mol}$ ], FPG $5.7-6.9 \mathrm{mmol} / 1$ or $2 \mathrm{~h}$ plasma glucose $7.8-11.0 \mathrm{mmol} / \mathrm{l}$ ); and [3] NGM (defined as $\mathrm{HbA}_{1 \mathrm{c}}<5.7 \%$ $[39 \mathrm{mmol} / \mathrm{mol}]$ and/or $\mathrm{FPG}<5.6 \mathrm{mmol} / \mathrm{l}$ and/or $2 \mathrm{~h}$ plasma glucose $<7.8 \mathrm{mmol} / \mathrm{l}$ ) [21].

Insulin and proinsulin levels were measured twice at an interval of 2 weeks.

Immunospecific insulin was measured in serum by a double-antibody radio immunoassay (lot SP21; Linco Research, St Louis, MO, USA), in which proinsulin and 32,33 split proinsulin cross-reacts by $0.2 \%$. Proinsulin was measured by a double-antibody radioimmunoassay (Lilly Laboratory for Clinical Research, Indianapolis, IN, USA), in which 31,32 proinsulin cross-reacts by $63 \%$. Serum total cholesterol, HDL-cholesterol and triacylglycerol were measured by enzymatic techniques (Boehringer-Mannheim, Mannheim, Germany) [20].

Insulin resistance was estimated by HOMA of insulin resistance (HOMA-IR) [22].

Cancer mortality rates follow-up The municipal register of the city of Hoorn supplied information on the vital status of all participants. Causes of death were derived from the medical records of general practitioners and from the local hospital. All causes of death were coded according to the International Classification of Diseases (ICD), Injuries and Causes of Death, ninth revision [23]. Death from cancer was defined as ICD-9 codes 140 to 239 (neoplasms). The mortality rate follow-up ended on 1 January 2009.

Statistical analysis Variables are presented as percentage, mean $( \pm \mathrm{SD})$ or median (interquartile range) in the event of a skewed distribution. Normality was tested for and if distribution was skewed, log-transformation was performed. Since, despite performance of log-transformation, proinsulin did not exhibit a linear relationship with cancer mortality 
rates, the population was categorised into tertiles of proinsulin levels. Differences in baseline characteristics between different tertiles of proinsulin level were tested with Student's $t$ tests (continuous variables) and $\chi^{2}$ tests (categorical variables). Differences in participants for whom proinsulin values were missing or not missing were tested using Student's $t$ tests (continuous variables) and $\chi^{2}$ tests (categorical variables).

Cumulative incidence rates of cancer and all-cause death were investigated according to tertiles of proinsulin. The follow-up duration was calculated as the time between baseline examination and date of death, loss to follow-up or end of follow-up on 1 January 2009.

Survival curves for tertiles of proinsulin were plotted for cancer and all-cause mortality. Proportional hazards assumptions for every model were tested by interpretation of the survival plots. Cox proportional hazards analyses were performed to investigate associations between proinsulin and cancer and all-cause mortality. First, we constructed a model in which we adjusted for age and sex. Then, we adjusted for possible confounding or mediating variables. The variables included are known to be associated with cancer (BMI and smoking) and/or are closely related to proinsulin (fasting specific insulin, estimated insulin resistance [HOMA-IR], glucose metabolism, fasting glucose).
We constructed two different models, one in which we adjusted for all possible confounding variables, and the second only adjusting for variables with values of $p<0.10$. To assess improvement of the model, changes in $-2 \log$ likelihood ( $-2 \mathrm{LL})$ were tested. We also tested for interaction with glucose metabolism (according to ADA 2011 criteria) and sex, by adding an interaction term to the model, where $p<0.10$ was considered to indicate statistically significant interactions. In a subpopulation $(n=189)$ for which we had 11-year follow-up information, sensitivity analyses were performed to investigate the possible mediating effect of the development of type 2 diabetes in the future and the possible use of glucose-lowering medication. Data are presented as HR with a $95 \%$ CI. The reported HRs can be interpreted as relative risks. Values of $p<0.05$ were considered statistically significant. All analyses were performed with SPSS 20 (SPSS, Chicago, IL, USA).

\section{Results}

Baseline characteristics Table 1 shows the baseline characteristics of participants according to different tertiles of proinsulin. None of the participants with type 2 diabetes
Table 1 Characteristics according to different tertiles of proinsulin concentration

Unless otherwise indicated, variables are presented as mean (SD) or median (interquartile range) in the event of skewed distribution

Number of missing values (if $>10$ missing): ${ }^{\mathrm{a}} 32 ;{ }^{\mathrm{b}} 38 ;{ }^{\mathrm{c}} 31$

${ }^{*} p<0.05$ (reference category proinsulin $<9.4 \mathrm{pmol} / \mathrm{l}$ )

\begin{tabular}{|c|c|c|c|}
\hline \multirow[t]{2}{*}{ Characteristic } & \multicolumn{3}{|c|}{ Tertile of proinsulin $(\mathrm{pmol} / \mathrm{l})$} \\
\hline & $<9.4$ & $9.4-16.5$ & $>16.5$ \\
\hline \multicolumn{4}{|l|}{ Baseline } \\
\hline$n$ & 146 & 146 & 146 \\
\hline Proinsulin (pmol/1) & $6.0(3.8,7.8)$ & $12.3(10.9,14.2)$ & $24.3(19.6,34.4)$ \\
\hline Age (years) & $63.9(7.5)$ & $64.0(7.1)$ & $65.0(6.8)$ \\
\hline Sex $(\%$ male $)$ & $69(47.3)$ & $60(41.1)$ & $89(61.1)^{*}$ \\
\hline Fasting glucose $(\mathrm{mmol} / \mathrm{l})$ & $5.7(1.1)$ & $5.9(0.9)$ & $6.7(1.8)^{*}$ \\
\hline $2 \mathrm{~h}$ postload glucose $(\mathrm{mmol} / \mathrm{l})$ & $7.2(3.4)$ & $8.2(3.0)^{*}$ & $10.4(4.5)^{*}$ \\
\hline $\mathrm{HbA}_{1 \mathrm{c}}(\%)$ & $5.5(0.8)$ & $5.5(0.6)$ & $5.9(1.0)^{*}$ \\
\hline $\mathrm{HbA}_{1 \mathrm{c}}(\mathrm{mmol} / \mathrm{mol})$ & 37 & 37 & $41^{*}$ \\
\hline $\operatorname{NGM}(n[\%])$ & $87(59.6)$ & $60(41.1)^{*}$ & $27(18.5)^{*}$ \\
\hline $\operatorname{IGM}(n[\%])$ & $41(28.1)$ & $57(39.0)^{*}$ & $54(37.0)^{*}$ \\
\hline Type 2 diabetes $(n[\%])$ & $18(12.3)$ & $29(19.9)^{*}$ & $65(44.5)^{*}$ \\
\hline Fasting specific insulin $(\mathrm{pmol} / \mathrm{l})$ & $74.6(55.4,92.5)$ & $81.6(63.2,112.2)^{*}$ & $120.6(88.9,159.7)^{*}$ \\
\hline HOMA-IR & $2.6(1.8,3.3)$ & $3.0(2.2,4.3)^{*}$ & $4.9(3.5,6.9)^{*}$ \\
\hline BMI $\left(\mathrm{kg} / \mathrm{m}^{2}\right)$ & $26.0(3.3)$ & $27.1(4.0)^{*}$ & $28.9(3.5)^{*}$ \\
\hline Physical activity (h per day) & $1.0(0.6,2.0)^{\mathrm{a}}$ & $1.1(0.5,1.8)^{\mathrm{b}}$ & $1.0(0.5,1.8)^{\mathrm{c}}$ \\
\hline Hypertension (\%) & $48(33.1)$ & $57(39.0)$ & $81(55.5)^{*}$ \\
\hline Current smoker (\%) & $40(29.4)$ & $35(24.3)$ & $32(21.9)$ \\
\hline Smoking (pack years) & $7.5(0.0,23.7)$ & $4.0(0.0,23.2)^{*}$ & $14.4(0.0,34.9)^{*}$ \\
\hline \multicolumn{4}{|l|}{20 year follow-up } \\
\hline All-cause mortality $(n[\%])$ & $61(41.8)$ & $52(35.6)$ & $83(56.8)^{*}$ \\
\hline Cancer mortality $(n[\%])$ & $14(9.6)$ & $13(8.9)$ & $26(17.8)^{*}$ \\
\hline
\end{tabular}


for whom proinsulin levels were available were using glucose-lowering medication. Compared with the lowest tertile (proinsulin $\leq 9.4 \mathrm{pmol} / \mathrm{l}$ ), participants with high levels of proinsulin ( $>16.5 \mathrm{pmol} / \mathrm{l})$ had significantly higher BMI, higher glucose levels, higher $\mathrm{HbA}_{1 \mathrm{c}}$ and higher fasting specific insulin concentrations. Within the highest tertile of proinsulin, 27 participants $(18.5 \%, 95 \%$ CI 12.7, 25.9) had NGM, $54(37.0 \%, 95 \%$ CI 29.3, 45.4) had IGM and 65 (44.5\%, 95\% CI 36.4, 52.9) had type 2 diabetes.

Participants who, for technical reasons $(n=63)$ or because of missing samples $(n=74)$, had missing proinsulin values had significantly lower fasting specific insulin values and lower HOMA-IR. This subpopulation also had fewer individuals with type 2 diabetes. The prevalence of cancer mortality was similar to that in the group for which proinsulin levels were available (12.9\% vs $13.1 \%)$.

Proinsulin and all-cause and cancer mortality rates The median duration of follow-up was 17.3 years. At the end of follow-up, 196 (44.7\%) of the 438 participants had died, 53 $(27 \%)$ of them from cancer. Individuals who died from cancer were older and had significantly higher specific insulin and proinsulin levels at baseline (data not shown). One third (31\%) of the participants who died from cancer had cancer as defined by ICD-9 codes 150 to 159 (malignant neoplasm of digestive organs and peritoneum). Other frequently present tumour groups were ICD-9 codes 235 to 238 (malignant neoplasm of uncertain origin [31\%]) and ICD-9 codes 160 to 165 (malignant neoplasm of respiratory and intrathoracic organs [19\%]) (data not shown). The all-cause mortality rate was highest in individuals with type 2 diabetes in each tertile of proinsulin. Figure 1 shows cancer mortality rates according to glucose metabolism (Fig. 1a) and stratified for tertiles of proinsulin (Fig. 1b). The cancer mortality rate was similar across the different groups of glucose metabolism and in the first two tertiles of proinsulin. The highest tertile of proinsulin $(>16.5 \mathrm{pmol} / \mathrm{l})$ had the highest cancer mortality rate, with NGM conferring the largest share.

The survival curves in Fig. 2 show that cancer mortality rates were significantly higher in the highest tertile of

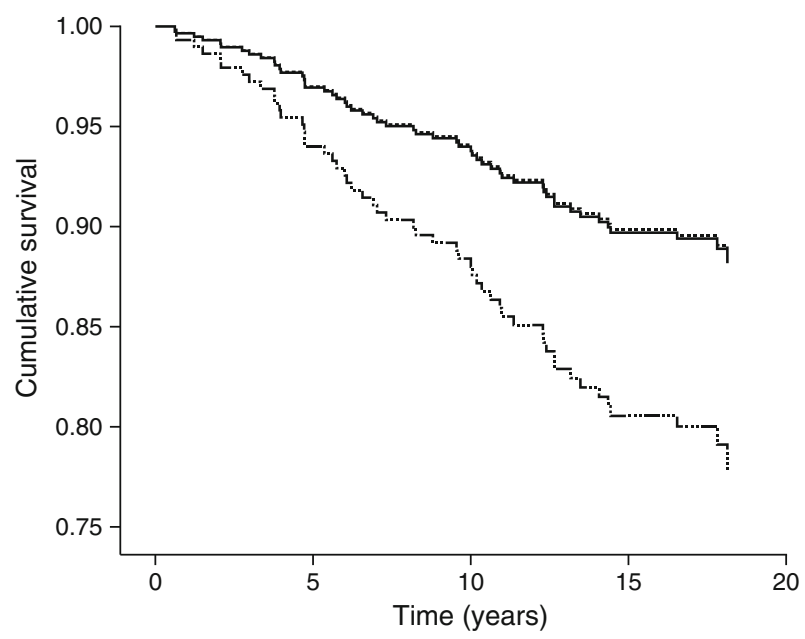

Fig. 2 Survival curves for the 20 year cancer mortality rate per tertiles of fasting proinsulin. Continuous line, $<9.4 \mathrm{pmol} / \mathrm{l}$; dotted line, 9.4 $16.5 \mathrm{pmol} / \mathrm{l}$; dashed and dotted line, $>16.5 \mathrm{pmol} / 1$

proinsulin $(>16.5 \mathrm{pmol} / \mathrm{l})$ than in the lowest $(<9.4 \mathrm{pmol} / \mathrm{l})$ and middle (9.4 to $16.5 \mathrm{pmol} / \mathrm{l}$ ) tertiles. No differences in HRs were observed between the first two tertiles and these were therefore combined and compared with the highest tertile $(>16.5 \mathrm{pmol} / \mathrm{l})$. Table 2 presents a Cox proportional hazards model for deaths from cancer, all causes and all causes except cancer. Proinsulin levels of $>16.5 \mathrm{pmol} / \mathrm{l}$ compared with levels $\leq 16.5 \mathrm{pmol} / 1$ were significantly associated with cancer mortality (HR 2.01, 95\% CI 1.16, 3.46). Adjustment for confounding showed a significant reduction of the -2LL, indicating a significant improvement of fit of the model. The association was influenced to some extent, but not entirely, by other risk factors. Glucose metabolism, BMI and smoking (pack years) were combined into a multivariate model, which did not significantly influence the association (HR 1.91, 95\% CI 1.04, 3.52). The highest tertile of proinsulin was associated with all-cause mortality rates (HR 1.6, 95\% CI 1.18, 2.24). After adjustment for potential confounders or mediating variables, the association diminished and did not remain significant. When cancer mortality rates were excluded from the model, we
Fig. 1 (a) Cancer mortality rates $(\%)$ according to different categories of glucose metabolism and (b) stratified for different tertiles of proinsulin. T2DM, type 2 diabetes
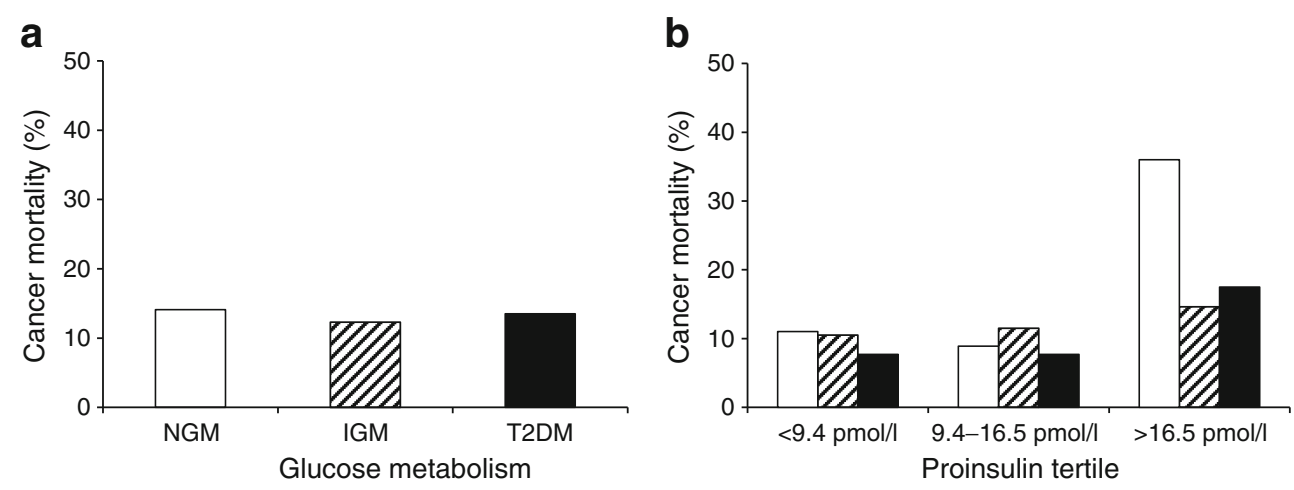
Table 2 Cancer, all-cause and all-cause except cancer mortality rates in the group with proinsulin $>16.5 \mathrm{pmol} / 1$ vs those with $\leq 16.5 \mathrm{pmol} / 1$ (reference category)

\begin{tabular}{|c|c|c|c|c|c|c|}
\hline & \multicolumn{6}{|c|}{ Mortality rates in group with proinsulin $>16.5 \mathrm{pmol} / 1$} \\
\hline & \multicolumn{2}{|l|}{ Cancer } & \multicolumn{2}{|l|}{ All-cause } & \multicolumn{2}{|c|}{ All causes except cancer } \\
\hline & $\operatorname{HR}(95 \% \mathrm{CI})$ & $-2 \mathrm{LL}$ & $\operatorname{HR}(95 \% \mathrm{CI})$ & $-2 \mathrm{LL}$ & $\operatorname{HR}(95 \% \mathrm{CI})$ & $-2 \mathrm{LL}$ \\
\hline Model 1 (adjusted for age and sex) & $2.01(1.16,3.46)^{*}$ & 593 & $1.62(1.18,2.24)^{*}$ & 1,693 & $1.45(0.97,2.17)$ & 1,092 \\
\hline $\begin{array}{l}\text { Model } 2 \text { (adjusted as for Model } 1+ \\
\text { glucose metabolism, BMI and smoking) }\end{array}$ & $1.91(1.04,3.52)^{*}$ & $584^{*}$ & $1.44(0.99,2.09)$ & $1,624^{*}$ & $1.24(0.78,1.98)$ & $1,027 *$ \\
\hline $\begin{array}{l}\text { Model } 3 \text { (adjusted as for Model 2+ } \\
\text { insulin and HOMA-IR) }\end{array}$ & $2.08(1.09,3.96)^{*}$ & $570^{*}$ & $1.47(0.99,2.16)$ & $1,595^{*}$ & $1.23(0.76,2.01)$ & $1,014^{*}$ \\
\hline
\end{tabular}

$* p<0.05$

observed an association between proinsulin $>16.5 \mathrm{pmol} / 1$ and all-cause mortality rates (HR 1.45 CI $0.97,2.17$ ) that did not reach statistical significance.

Additional analyses We tested for interaction of proinsulin with glucose metabolism and sex, by adding interaction terms into the crude model. Both product terms for glucose metabolism (IGM vs NGM, $p=0.11$ and type 2 diabetes vs NGM, $p=0.67)$ and sex $(p=0.21)$ were not statistically significant. We also stratified the analyses to visually examine whether the effect was different for different categories of glucose metabolism, and although power was weaker due to the smaller groups, the association between proinsulin and cancer mortality rates was almost equal in every category of glucose metabolism.

\section{Discussion}

The present study shows that high proinsulin $(>16.5 \mathrm{pmol} / \mathrm{l})$ levels are significantly associated with a twofold risk of cancer mortality over a 20 year period compared with proinsulin levels below $16.5 \mathrm{pmol} / \mathrm{l}$. This association was independent of glucose metabolism, fasting glucose, specific insulin, estimated insulin resistance, BMI and smoking (Table 2). Furthermore, this study shows that proinsulin levels were more strongly associated with cancer mortality than with all-cause mortality rates.

To our knowledge, this is the first paper to report on the association between proinsulin and cancer mortality rates. The in vitro study of Malaguarnera et al [14] might provide a possible mechanistic link for our findings, showing as it did that proinsulin differentially binds to and activates the two insulin receptor isoforms, with a higher affinity for IR-A than for insulin receptor $\mathrm{B}$ (IR-B). IR-A is a low-specificity receptor with high affinity not only for insulin but also for IGF-II; it can also activate intracellular signalling and biological effects in response to IGF-I. The above study [14] also showed that proinsulin was almost equipotent to insulin in inducing cell proliferation and migration in cells expressing IR-A at various levels.

As in other studies [12, 24], proinsulin levels were associated with specific insulin levels and with insulin resistance (Table 1). Therefore, fasting specific insulin could have mediated the association between proinsulin and cancer mortality rates. However, adjustment for specific insulin in the Cox regression analysis did not diminish the statistically significant association between proinsulin and cancer mortality rates.

The association between proinsulin levels and cancer mortality rates did not significantly differ across different categories of glucose metabolism (Fig. 1). It is possible that individuals with NGM and high proinsulin levels may have developed type 2 diabetes in the future (i.e. post baseline), which might then have mediated the association between high proinsulin and cancer mortality rates in individuals with NGM. For almost half $(n=189)$ of our study population, we had access to information about glucose metabolism 11 years later. Of that group, ten (11.2\%) of 89 individuals with NGM had developed type 2 diabetes and only one of these ten died from cancer. Of the 69 persons with IGM for whom we had follow-up information, 37 (53.6\%) developed type 2 diabetes 11 years later, none of whom died from cancer during the 20 year follow-up period. Therefore, it is unlikely that the development of type 2 diabetes in the future (i.e. post baseline) would explain our results. At baseline, none of the participants with type 2 diabetes in our study population was using glucose-lowering medication, which made it possible to research the association between proinsulin and cancer mortality rates. It is also possible that the use of glucose-lowering medication post baseline could have biased our results. Therefore sensitivity analyses were performed and showed that in the group for whom we had follow-up information $(n=189), 36$ participants used glucose-lowering medication 11 years later, of whom three died from cancer.

The strengths of this study include the long and complete follow-up duration (none of the participants in this 
subsample were lost to follow-up) with a precise causespecific mortality registry. This enabled us to study a more precise association between proinsulin and cancer mortality rates. Furthermore, the Hoorn Study consists of a randomly selected cohort population, which limits the possibility of selection bias. Another strength of our study is that fasting proinsulin and specific insulin were measured at baseline, in duplicate, which made it possible to evaluate whether the association between proinsulin and cancer mortality rates was independent of specific insulin. A high correlation between proinsulin and specific insulin could cause multicollinearity within the regression model. We observed a Pearson correlation coefficient between proinsulin and specific insulin of 0.48 and the regression model did not substantially change when specific insulin was added to the model (Table 2).

A limitation of our study is that the radioimmunoassay used was not able to differentiate between intact proinsulin and des 31,32 proinsulin. The inhibition of the conversion from des 31,32 proinsulin to insulin by hyperglycaemia [11] could therefore, to some extent, lead to an overestimation of the contribution of proinsulin levels. Proinsulin concentrations are highly variable. We therefore measured fasting proinsulin levels on two separate days, at an interval of 2 weeks, and used the mean to improve precision. Furthermore, proinsulin levels were only measured at baseline and there is no indication that proinsulin levels remained in the same tertile during the complete follow-up period. Therefore we can only draw conclusions on the association between proinsulin measured at baseline and cancer mortality rates in a 20 year follow-up period. Another restriction is the relatively small sample size of this study. However, the small sample size did not result in wide confidence intervals, indicating that an apparent association between proinsulin and cancer mortality rates does indeed exist. Due to the small study population, we lacked power to differentiate between site-specific cancer mortality rates, so we can only draw conclusions on the association between proinsulin and overall cancer mortality rates. In addition, confounding might have affected our analysis. Although we adjusted for several confounders at baseline (including specific insulin), 20 years elapsed between measurements and outcomes. Since we did not adjust for these potential confounders during the follow-up period, it remains possible that other variables at follow-up might explain part of our observations.

To conclude, high proinsulin levels were found to be significantly associated with 20 year cancer mortality, independently of glucose metabolism and fasting specific insulin. These findings need to be reproduced in other populations, but are in line with existing in vitro findings [14]. Additional studies with site-specific cancer information are warranted.
Acknowledgements I. Walraven is the guarantor of this work and, as such, had full access to all the data in the study and takes responsibility for the integrity of the data and the accuracy of the data analysis

Duality of interest The authors declare that there is no duality of interest associated with the manuscript.

Contribution statement Author contributions were as follows: JMD, GN and CDAS were responsible for the conception and design of the study; IW and GN analysed the data and wrote the paper. All authors contributed to the interpretation of data and critically reviewed the manuscript. All authors approved the final version submitted for publication.

\section{References}

1. Yang XL, Ma RCW, Chan JCN (2011) Meta-analysis of trial data may support a causal role of hyperglycaemia in cancer. Diabetologia 54:709-710

2. Yang X, Ko GTC, So WY et al (2010) Associations of hyperglycemia and insulin usage with the risk of cancer in type 2 diabetes: The Hong Kong Diabetes Registry. Diabetes 59:1254-1260

3. Coughlin SS, Calle EE, Teras LR, Petrelli J, Thun MJ (2004) Diabetes mellitus as a predictor of cancer mortality in a large cohort of US adults. Am J Epidemiol 159:1160-1167

4. Rapp K, Schroeder J, Klenk J et al (2006) Fasting blood glucose and cancer risk in a cohort of more than 140,000 adults in Austria. Diabetologia 49:945-952

5. Harding JL, Soderberg S, Shaw JE et al (2012) All-cause cancer mortality over 15 years in multi-ethnic Mauritius: the impact of diabetes and intermediate forms of glucose tolerance. Int J Cancer 131:2385-2393

6. Saydah SH, Loria CM, Eberhardt MS, Brancati FL (2003) Abnormal glucose tolerance and the risk of cancer death in the United States. Am J Epidemiol 157:1092-1100

7. Zhou X, Qiao Q, Zethelius B et al (2010) Diabetes, prediabetes and cancer mortality. Diabetologia 53:1867-1876

8. Pollak M (2008) Insulin, insulin-like growth factors and neoplasia. Best Practice \& Research Clinical Endocrinology \& Metabolism 22:625-638

9. Larsson H, Ahrén B (1999) Relative hyperproinsulinemia as a sign of islet dysfunction in women with impaired glucose tolerance. J Clin Endocrinol Metab 84:2068-2074

10. Mykkänen L, Haffner S, Kuusisto J, Pyörälä K, Hales C, Laakso M (1995) Serum proinsulin levels are disproportionately increased in elderly prediabetic subjects. Diabetologia 38:1176-1182

11. Nijpels G, Popp-Snijders C, Kostense PJ, Bouter LM, Heine RJ (1996) Fasting proinsulin and 2-h post-load glucose levels predict the conversion to NIDDM in subjects with impaired glucose tolerance: the Hoorn Study. Diabetologia 39:113-118

12. Pfützner A, Kunt T, Hohberg C et al (2004) Fasting intact proinsulin is a highly specific predictor of insulin resistance in type 2 diabetes. Diabetes Care 27:682-687

13. Wareham NJ, Byrne CD, Williams R, Day NE, Hales CN (1999) Fasting proinsulin concentrations predict the development of type 2 diabetes. Diabetes Care 22:262-270

14. Malaguarnera R, Sacco A, Voci C, Pandini G, Vigneri R, Belfiore A (2012) Proinsulin binds with high affinity the insulin receptor isoform $\mathrm{A}$ and predominantly activates the mitogenic pathway. Endocrinology 153:2152-2163 
15. Frasca F, Pandini G, Scalia P et al (1999) Insulin receptor isoform A, a newly recognized, high-affinity insulin-like growth factor II receptor in fetal and cancer cells. Mol Cell Biol 19:3278-3288

16. Vella V, Pandini G, Sciacca L et al (2002) A novel autocrine loop involving IGF-II and the insulin receptor isoform-A stimulates growth of thyroid cancer. J Clin Endocrinol Metab 87:245-254

17. Belfiore A (2007) The role of insulin receptor isoforms and hybrid insulin/IGF-I receptors in human cancer. Curr Pharm Des 13:671-686

18. Mooy JM, Grootenhuis PA, de Vries H et al (1995) Prevalence and determinants of glucose intolerance in a Dutch Caucasian population. The Hoorn Study. Diabetes Care 18:1270-1273

19. Alssema M, Dekker JM, Nijpels G, Stehouwer CDA, Bouter LM, Heine RJ (2005) Proinsulin concentration is an independent predictor of all-cause and cardiovascular mortality. Diabetes Care 28:860-865
20. Mooy JM, Grootenhuis PA, de Vries H et al (1996) Intra-individual variation of glucose, specific insulin and proinsulin concentrations measured by two oral glucose tolerance tests in a general Caucasian population: the Hoorn Study. Diabetologia 39:298-305

21. American Diabetes Association (2011) Diagnosis and classification of diabetes mellitus. Diabetes Care 34(Suppl 1):S62-S69

22. Matthews DR, Hosker JP, Rudenski AS et al (1985) Homeostasis model assessment: insulin resistance and beta-cell function from fasting plasma glucose and insulin concentrations in man. Diabetologia 28:412-419

23. Kupka K (1978) International classification of diseases: ninth revision. WHO Chron 32:219-225

24. Haffner SM, Bowsher RR, Mykkanen L et al (1994) Proinsulin and specific insulin concentration in high- and low-risk populations for NIDDM. Diabetes 43:1490-1493 